\title{
Iatrogenic left main coronary artery dissection
}

\author{
K. Onsea $\cdot$ P. Kayaert $\cdot$ W. Desmet $\cdot$ C. L. Dubois
}

Published online: 8 February 2011

(C) Springer Media / Bohn Stafleu van Loghum 2011

\begin{abstract}
We present a case of iatrogenic left main coronary artery dissection, successfully treated by prompt bail-out stenting, and provide a brief discussion on its occurrence and treatment, as well as the immediate and long-term outcome of percutaneous coronary intervention, including our own single-centre experience, for this potentially catastrophic complication.
\end{abstract}

Keywords Left main coronary artery Dissection .

Percutaneous intervention

\section{Introduction}

Iatrogenic left main coronary artery (LMCA) dissection is one of the complications most dreaded by the interventional cardiologist. Although rare, with a reported incidence of less than $0.1 \%$ [1], its occurrence can have devastating consequences if not promptly treated with immediate revascularisation. LMCA dissection often leads to abrupt vessel closure and cessation of blood flow towards a large portion of myocardium resulting in acute pump failure and haemodynamic collapse. Before 1993, when the first successful percutaneous bail-out LMCA stenting was performed, urgent coronary artery bypass surgery (CABG) was the treatment of choice. However, many patients died before even making it to the operation theatre. Hence immediate percutaneous coronary intervention (PCI) seems to be an appropriate and feasible alternative if performed by an experienced interventionalist, as demonstrated by the next case.

K. Onsea $(\bowtie) \cdot$ P. Kayaert $\cdot$ W. Desmet $\cdot$ C. L. Dubois Department of Cardiology, University Hospital Leuven, Herestraat 49,

3000 Leuven, Belgium

e-mail: kevin.onsea@uzleuven.be

\section{Case}

A 74-year old woman with evidence of silent anterior myocardial ischaemia on non-invasive stress testing was referred for coronary angiography, which showed singlevessel disease with a significant lesion in the mid-part of the left anterior descending coronary artery (LAD) (Fig. 1, Panels $a+b$ ). Because the lesion had a heavily calcified appearance, the patient was scheduled for elective PCI with a plan to perform rotational atherectomy for plaque debulking prior to stent deployment. A right femoral approach was chosen with insertion of a $6 \mathrm{~F}$ introducer sheath and an Extra Back-Up 3.75 guiding catheter $\left(\right.$ Medtronic $^{\circledR}$ ) was used. Engagement of the LMCA proved rather difficult due to its superior location but was successfully established after several attempts. The pressure curve remained normal throughout without damping or ventricularisation. However, upon first injection of contrast dye, an antegrade dissection was visualised 'as it happened', extending from the proximal to the distal LMCA (Fig. 2, Panels $\mathrm{c}+\mathrm{d}$ ), which abruptly halted coronary flow, almost immediately resulting in cardiogenic shock. Treatment with vasopressors, inotropes and artificial ventilation was promptly initiated and an intra-aortic balloon pump (IABP) was inserted via the contralateral groin. Nevertheless, the haemodynamic condition of the patient remained so precarious that we thought she would not survive any further delay while awaiting emergent surgery. Therefore we opted for percutaneous treatment. We were able to carefully negotiate a wire in the true lumen of the LMCA and a bare metal stent (Vision $3.5 \times 28 \mathrm{~mm}$, Abbott Vascular ${ }^{\circledR}$ ) was subsequently placed from the ostial LMCA towards the LAD (Fig. 3, Panel e), re-establishing TIMI 3 flow in both the LAD and the circumflex artery (Fig. 3, panel f). 
Fig. 1 Coronary angiogram in left anterior oblique cranial (panel a) and right anterior oblique cranial (panel b) view showing a severe, highly calcified lesion (arrow) in the middle part of the left anterior descending artery
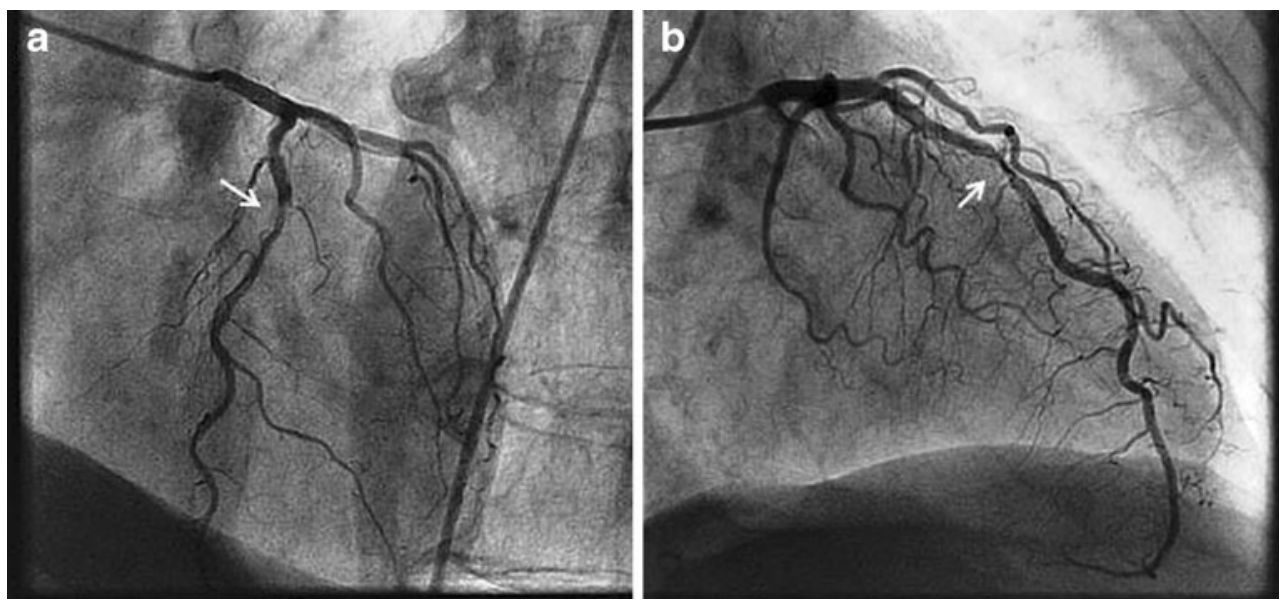

The angiographic result was further optimised using noncompliant balloon post-dilatations (Sprinter NC 3.75 $\times 15 \mathrm{~mm}$, Medtronic $^{\circledR}$ ), after which the patient was transferred to the intensive care unit for further monitoring. Vasopressive and inotropic agents could quickly be titrated down and stopped, and the IABP was removed the next day. Extubation had to be delayed due to aspiration pneumonia, but after 10 days the patient was discharged home in a good overall condition. Predischarge echocardiography revealed a normal systolic left ventricular function, and maximum troponin-I elevation during the entire hospital course was $0.49 \mu \mathrm{g} / 1$ (upper limit of normal: $0.13 \mu \mathrm{g} / \mathrm{l}$ ).

\section{Discussion}

Iatrogenic LMCA dissection results from mechanical injury to the arterial wall during catheter manipulation or passage or deployment of an interventional device. Extensive catheter manipulation, catheter type (e.g. Amplatz catheter, small Judkins catheter resulting in deep LMCA intubation), stiffer and less manageable guide wires (e.g. pressure wire), unusual LMCA anatomy or location, operator experience, and presence of LMCA atherosclerosis have all been associated with an increased risk of dissection $[2,3]$.

In our case, the introduction of the catheter in the LMCA was more difficult than expected due to its high location. Although no damping or ventricularisation of the pressure curve was seen, the tip of the catheter was 'scraping' against the wall of the LMCA, thereby probably causing the dissection when injecting dye. Hence, the importance of careful positioning of the catheter in co-axial alignment with the artery before any vigorous contrast injection cannot be stressed enough, especially when confronted with an already calcified LMCA.

Once dissection occurs, the clinical picture differs, depending on the remaining antegrade coronary flow, and can range from an asymptomatic patient with preserved TIMI 3 flow, to a patient in refractory cardiogenic shock whose LMCA is 'amputated'. Nevertheless, even in case of initial TIMI 3 flow and haemodynamic stability, rapid deterioration may occur shortly after because of abrupt flow compromise due to progressive dissection or superimposed thrombus formation, which is why it seems imperative to always envisage a 'revascularisation plan' by $\mathrm{PCI}$ or CABG.
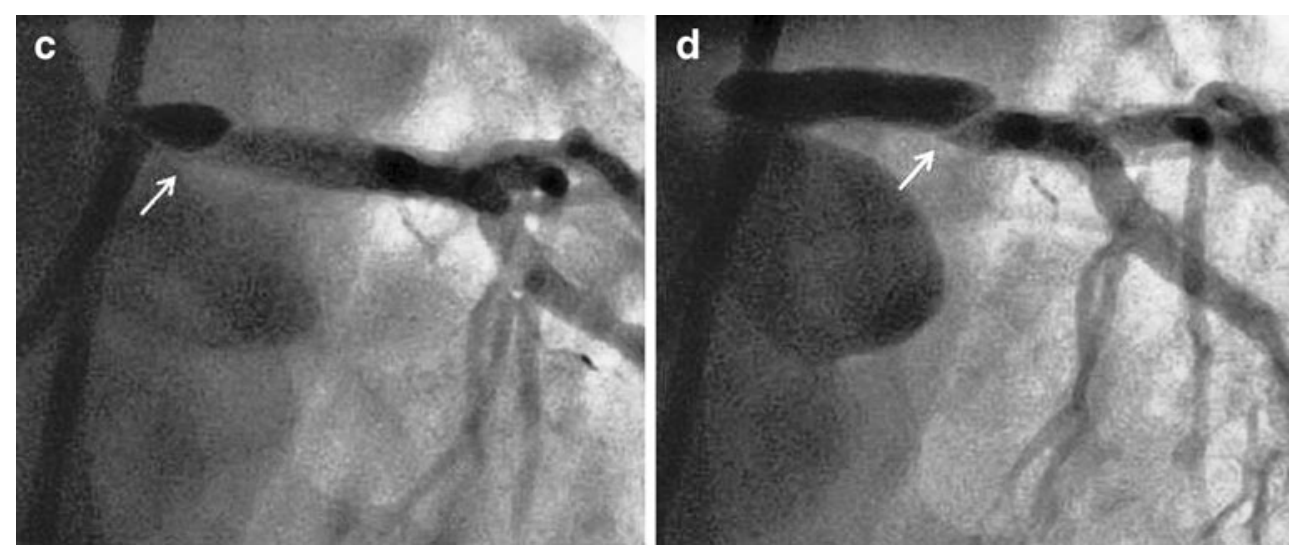

Fig. 2 Iatrogenic dissection (arrow) upon dye injection extending from the proximal (panel c) to distal (panel d) left main coronary artery 
Fig. 3 Deployment of a $3.5 \times$ $28 \mathrm{~mm}$ bare metal stent from the ostial left main coronary artery towards the proximal left anterior descending artery (panel e), sealing the dissection and re-establishing TIMI 3 flow (panel f)
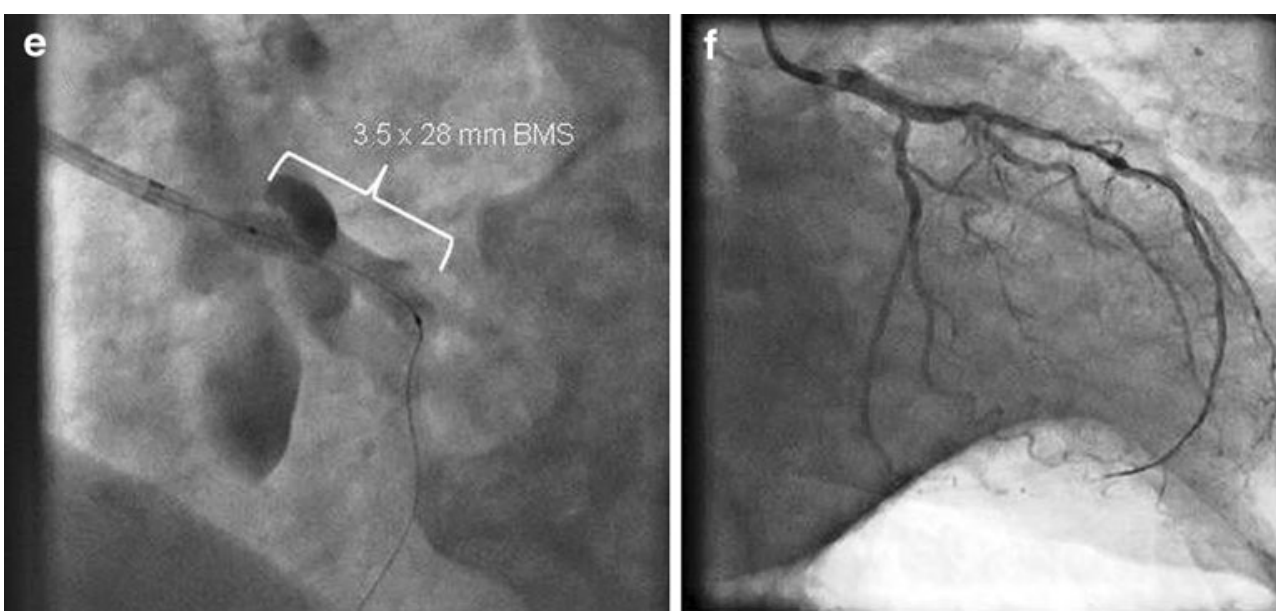

A literature review by Cheng et al. [4] (Table 1) of 36 patients who underwent stenting of an iatrogenic LMCA dissection showed a favourable immediate outcome with achievement of procedural, angiographic success in 32 patients (88.9\%). Four patients needed emergent CABG of whom 2 eventually died. This strategy, consisting of a primary attempt to stent with back-up CABG when needed, thus resulted in an overall survival rate of $94.4 \%$.

Long-term outcome of emergent LMCA bail-out stenting in a group of 13 patients [4] was similar to that of elective percutaneous treatment of an unprotected LMCA, with a binary restenosis rate of $30 \%$ of the LMCA segment (3 patients) at $5 \pm 2$ months follow-up. In another report, Lee and colleagues [5] reported no significant LMCA restenosis on 6-month follow-up angiogram of ten urgently treated patients, which was attributed to the minimal plaque burden in the iatrogenically dissected LMCA, leading to less in-stent neo-intimal proliferation.

In our own hospital - a tertiary reference centre-we have treated 18 patients for iatrogenic LMCA dissection since 1998. Immediate procedural success was $100 \%$ with a 1-year survival rate of $89 \%$ (16/18 patients). Of the two patients who died, one succumbed shortly after successful PCI due to uncontrollable lung bleeding, while the other patient died from lung cancer.

Follow-up angiogram at 6 months was performed in eight patients, one of whom showed significant restenosis at the LMCA stent necessitating subsequent CABG. Early stent thrombosis occurred in one patient 10 days after LMCA stenting, while being hospitalised for upper gastrointestinal bleeding interfering with normal dual antiplatelet therapy. Unfortunately emergent percutaneous treatment was unsuccessful in this patient, who subsequently underwent $\mathrm{CABG}$, but eventually required a heart transplant because of persistent severe pump failure.

\section{Conclusion}

When confronted with an iatrogenic LMCA dissection, prompt bail-out stenting seems a reasonable and feasible first-choice option, with high immediate procedural success and acceptable long-term results. As always, prevention is better than cure and meticulous attention should be paid to
Table 1 Literature review of iatrogenic LMCA dissection: review by Cheng [4], completed by cases from our own experience $(n=18)$ since 1998

\footnotetext{
${ }^{\mathrm{a}}$ One patient died of pulmonary haemorrhage, the other of lung cancer
}

\begin{tabular}{lllll}
\hline 1st author & Case number & Successful bail-out stenting & Emergent CABG & Survival \\
\hline Garcia-Robles JA [6] & 1 & 0 & 1 & 1 \\
Hennessy TG [7] & 1 & 1 & 0 & 1 \\
Cameron J [8] & 6 & 6 & 0 & 6 \\
Al-Saif SM [9] & 1 & 1 & 0 & 1 \\
Nageh T [10] & 1 & 1 & 0 & 1 \\
Jain D [11] & 1 & 1 & 0 & 1 \\
Mulvihill NT [12] & 1 & 1 & 0 & 1 \\
Awadalla H [13] & 1 & 0 & 1 & 10 \\
Lee SW [5] & 10 & 10 & 0 & 11 \\
Cheng Cl [4] & 13 & 11 & 2 & $16^{\mathrm{a}}$ \\
Onsea K & 18 & 18 & 0 & 51 \\
Total & 54 & 50 & 4 & 10 \\
\hline
\end{tabular}


catheter and device manipulation during each diagnostic or interventional procedure.

\section{References}

1. Awadalla H, Sabet S, El Sebaie A, et al. Catheter-induced left main dissection incidence, predisposition and therapeutic strategies experience from two sides of the hemisphere. J Invasive Cardiol. 2005;17 (4):233-6.

2. Kovac JD, de Bono DP. Cardiac catheter complications related to left main stem disease. Int J Cardiol. 1999;69(3):299-303.

3. Slack JD, Pinkerton CA, VanTassel JW, et al. Left main coronary artery dissection during percutaneous transluminal coronary angioplasty. Cathet Cardiovasc Diagn. 1986;12(4):255-60.

4. Cheng $\mathrm{CI}, \mathrm{Wu} \mathrm{CJ}$, Hsieh YK, et al. Percutaneous coronary intervention for iatrogenic left main coronary artery dissection. Int $\mathrm{J}$ Cardiol. 2008;126(2):177-82.

5. Lee SW, Hong MK, Kim YH, et al. Bail-out stenting for left main coronary artery dissection during catheter-based procedure: acute and long-term results. Clin Cardiol. 2004;27(7):393-5.

6. Garcia-Robles JA, Garcia E, Rico M, et al. Emergency coronary stenting for acute occlusive dissection of the left main coronary artery. Cathet Cardiovasc Diagn. 1993;30(3):227-9.
7. Hennessy TG, McCann HA, Sugrue DD. Bailout Palmaz-Schatz stenting for iatrogenic dissection of the left main coronary artery. J Invasive Cardiol. 1996;8:450-2.

8. Cameron J, Aroney C, Bett J, et al. Left main coronary artery dissection during coronary angioplasty or angiography treated by stent insertion without requirement for emergency bypass graft surgery. Aust N Z J Med. 2000;30(6):726-8.

9. Al-Saif SM, Liu MW, Al-Mubarak N, et al. Percutaneous treatment of catheter-induced dissection of the left main coronary artery and adjacent aortic wall: a case report. Catheter Cardiovasc Interv. 2000;49(1):86-9.

10. Nageh T, Badawi R, Thomas MR, et al. Left main coronary artery dissection detected by intravascular ultrasound following angiographically successful percutaneous coronary intervention. J Invasive Cardiol. 2004;16(5):279-80.

11. Jain D, Kurowski V, Katus HA, et al. Catheter-induced dissection of the left main coronary artery, the nemesis of an invasive cardiologist a case report and review of the literature. Z Kardiol. 2002;91(10):840-5.

12. Mulvihill NT, Boccalatte M, Fajadet J, et al. Catheter-induced left main dissection: a treatment dilemma. Catheter Cardiovasc Interv. 2003;59(2):214-6.

13. Awadalla H, Salloum JG, Smalling RW, et al. Catheter-induced dissection of the left main coronary artery with and without extension to the aortic root: a report of two cases and a review of the literature. J Interv Cardiol. 2004;17(4):253-7. 